\title{
Genome-wide identification of RNA editing in seven porcine tissues by matched DNA and RNA high-throughput sequencing
}

Yuebo Zhang, Longchao Zhang ${ }^{*}$, Jingwei Yue, Xia Wei, Ligang Wang, Xin Liu, Hongmei Gao, Xinhua Hou, Fuping Zhao, Hua Yan and Lixian Wang ${ }^{*}$

\begin{abstract}
Background: RNA editing is a co/posttranscriptional modification mechanism that increases the diversity of transcripts, with potential functional consequences. The advent of next-generation sequencing technologies has enabled the identification of RNA edits at unprecedented throughput and resolution. However, our knowledge of RNA editing in swine is still limited.

Results: Here, we utilized RES-Scanner to identify RNA editing sites in the brain, subcutaneous fat, heart, liver, muscle, lung and ovary in three 180-day-old Large White gilts based on matched strand-specific RNA sequencing and whole-genome resequencing datasets. In total, we identified 74863 editing sites, and $92.1 \%$ of these sites caused adenosine-to-guanosine (A-to-G) conversion. Most A-to-G sites were located in noncoding regions and generally had low editing levels. In total, 151 A-to-G sites were detected in coding regions (CDS), including 94 sites that could lead to nonsynonymous amino acid changes. We provide further evidence supporting a previous observation that pig transcriptomes are highly editable at PRE-1 elements. The number of A-to-G editing sites ranged from 4155 (muscle) to 25001 (brain) across the seven tissues. The expression levels of the ADAR enzymes could explain some but not all of this variation across tissues. The functional analysis of the genes with tissuespecific editing sites in each tissue revealed that RNA editing might play important roles in tissue function. Specifically, more pathways showed significant enrichment in the fat and liver than in other tissues, while no pathway was enriched in the muscle.

Conclusions: This study identified a total of 74863 nonredundant RNA editing sites in seven tissues and revealed the potential importance of RNA editing in tissue function. Our findings largely extend the porcine editome and enhance our understanding of RNA editing in swine.
\end{abstract}

Keywords: ADAR, A-to-G, High-throughput sequencing, RNA editing, Swine

\section{Background}

In the 1980s, Benne et al. discovered an RNA editing event in which four nonencoded nucleotides were inserted into the mRNA of the mitochondrial cytochrome oxidase subunit II (coxII) gene in trypanosomatids [1]. Subsequently, RNA editing was defined as a co/ posttranscriptional modification mechanism that alters sequence information at the RNA level by introducing differences between a final RNA sequence and its

\footnotetext{
* Correspondence: zhlchias@163.com; iaswlx@263.net

Key Laboratory of Animal (Poultry) Genetics Breeding and Reproduction, Ministry of Agriculture; Institute of Animal Science, Chinese Academy of Agricultural Sciences, Beijing 100193, China
}

template DNA through the insertion, deletion or substitution of nucleotides [2]. This modification can occur in coding regions (CDS) and noncoding regions, thereby recoding amino acids, affecting alternative splicing, influencing RNA stability, and modulating the nuclear retention of RNAs [3, 4]. In mammals, adenosine-toinosine (A-to-I) editing catalyzed by adenosine deaminases acting on RNA (ADAR) is the most common type of RNA editing $[5,6]$. As inosine is generally read as guanosine (G) by the cellular machinery, A-to-I editing is also named A-to-G editing. The ADAR enzyme family, which primarily includes three members (ADAR1,

(c) The Author(s). 2019 Open Access This article is distributed under the terms of the Creative Commons Attribution 4.0 International License (http://creativecommons.org/licenses/by/4.0/), which permits unrestricted use, distribution, and reproduction in any medium, provided you give appropriate credit to the original author(s) and the source, provide a link to the Creative Commons license, and indicate if changes were made. The Creative Commons Public Domain Dedication waiver (http://creativecommons.org/publicdomain/zero/1.0/) applies to the data made available in this article, unless otherwise stated. 
ADAR2 and ADAR3), targets only double-stranded RNA to catalyze A-to-G RNA editing [7]. Both ADAR1 and ADAR2 are essential for normal development, and a homozygous null mutation in either of these two genes causes early lethality in mice $[8,9]$.

The identification of RNA editing sites heavily depends on sequencing technologies. Therefore, RNA edits were originally regarded as rare variants due to the limitations of sequencing technologies [10]. The advent of next-generation sequencing (NGS) technologies has enabled the identification of transcriptome-wide RNA editing events across individuals and tissues at unprecedented throughput and resolution. Subsequently, the discovery rate of RNA editing sites has dramatically increased. With the development of bioinformatics tools designed for RNA editing detection and the significant decline in sequencing costs, RNA editing studies are ushering in an unprecedented opportunity. Currently, RNA editing studies are widely implemented in humans and mice by comparing matched RNA and DNA sequencing data or using only RNA sequencing (RNA-seq) data [11-19]. Moreover, separate laboratories have reported the potentially biological significance of RNA editing in disease pathobiology and tumorigenicity $[20,21]$, introducing a new regulatory layer to enhance our understanding of complex diseases. Pigs are considered an ideal animal model of human diseases since they share similar anatomic and physiologic characteristics with humans. Therefore, RNA editing studies involving pigs can promote an understanding of the molecular basis of human diseases. However, to the best of our knowledge, only one research study, which identified 5294 nonredundant A-to-G sites across three tissues from one pig, has provided information about RNA editing at a transcriptome-wide level in pigs [22]. Our knowledge of RNA editing in pigs is very limited compared to that in humans and other model species.

Given the significance of pigs in biomedical research and animal husbandry, we systematically detected and characterized the RNA editome in pigs based on strand-specific RNA sequencing data and whole-genome sequencing data of the brain, fat, heart, liver, lung, muscle and ovary from three 180-day-old Large White gilts. We revealed a total of 74863 RNA editing sites and implemented a detailed characterization of the sequence and distribution features of these sites. We found frequent occurrence in noncoding regions, especially PRE-1, providing further evidence supporting a previous observation that pig transcriptomes are highly editable in PRE-1 elements. Furthermore, the functional analysis of the genes with tissue-specific editing sites in each tissue revealed the potential functional importance of RNA editing in porcine tissue regulation. Our study largely extends the list of RNA editing sites in swine and provides deeper insight into the characteristics of the pig editome.

\section{Methods}

\section{Sample collection and nucleic acid isolation}

The frontal lobe of the brain, tip of the heart, left lateral lobe of the liver, caudal lobe of the left lung, Longissimus dorsi (the $10-11^{\text {th }}$ rib, muscle), follicles and surrounding tissue of the ovary, and shoulder subcutaneous fat were collected from three 180-day-old Large White gilts (Table 1 and Additional file 1: Figure S1), snap frozen in liquid nitrogen and stored at $-80^{\circ} \mathrm{C}$ until use. The genomic DNA was extracted from the muscle samples by using the standard phenol-chloroform protocol. The total RNA was isolated using TRIzol reagent (Invitrogen, Carlsbad, CA, USA) according to the manufacturer's instructions. Only DNA samples with OD 260/280 ratios of $1.8 \sim 2.0$ and total contents greater than $1.5 \mu \mathrm{g}$ were used in the subsequent steps. RNA samples with RNA integrity number (RIN) scores higher than seven were used in this study.

\section{Strand-specific transcriptome sequencing}

Three micrograms of total RNA per sample were used for the subsequent creation of strand-specific RNA-seq libraries. In total, 21 strand-specific sequencing libraries were generated using mRNA purified from total RNA using oligo(dT) beads by NEBNext ${ }^{\circ}$ Ultra $^{\mathrm{T}}$ Directional RNA Library Prep Kit for Illumina ${ }^{\circ}$ (NEB, USA) following the manufacturer's protocol. After qualification by an Agilent 2100 Bioanalyzer and real-time PCR, each library preparation was sequenced on an Illumina HiSeq platform by the Novogene Bioinformatics Technology Cooperation (Beijing, China), and 150 bp paired-end reads were generated.

\section{Whole-genome sequencing}

Muscle DNA was extracted from the three pigs mentioned above and used to construct three DNA libraries (Additional file 1: Figure S1). These libraries were generated using a TruSeq Nano DNA HT Sample Preparation Kit (Illumina, USA) according to the manufacturer's recommendations. The quantification and quality of the sequencing libraries were assessed by real-time PCR and an Agilent Bioanalyzer 2100 system. Then, each library constructed above was sequenced on an Illumina HiSeq platform provided by the Novogene Bioinformatics Technology Cooperation (Beijing, China), and $150 \mathrm{bp}$ paired-end reads were generated for further analysis.

\section{Read processing}

To ensure the reliability of the reads and reduce the inherent noise of high-throughput sequencing in further analysis, the raw data were first filtered by eliminating the reads containing an adapter or poly- $\mathrm{N}$ and low-quality reads through a series of in-house Perl scripts used for quality control (QC). All downstream analyses were based on high-quality filtered data. 
Table 1 Statistics of the high-throughput sequencing

\begin{tabular}{|c|c|c|c|c|c|c|c|}
\hline \multirow[t]{2}{*}{ Sample name } & \multirow[t]{2}{*}{ Tissue type } & \multirow[t]{2}{*}{ Individual } & \multicolumn{2}{|l|}{ RNA-seq } & \multicolumn{3}{|l|}{ DNA-seq } \\
\hline & & & Total reads & $\overline{\text { Total mapped rate }}$ & Total reads & Mapping rate & Coverage $^{a}$ \\
\hline Brain1 & Brain & 1 & $65,772,910$ & $86.7 \%$ & & & \\
\hline Brain2 & Brain & 2 & $77,952,110$ & $87.9 \%$ & & & \\
\hline Brain3 & Brain & 3 & $102,517,764$ & $87.9 \%$ & & & \\
\hline Fat1 & Fat & 1 & $71,195,080$ & $83.9 \%$ & & & \\
\hline Fat2 & Fat & 2 & $74,251,406$ & $85.2 \%$ & & & \\
\hline Fat3 & Fat & 3 & $69,499,032$ & $85.0 \%$ & & & \\
\hline Heart1 & Heart & 1 & $69,228,312$ & $88.1 \%$ & & & \\
\hline Heart2 & Heart & 2 & $74,663,108$ & $87.0 \%$ & & & \\
\hline Heart3 & Heart & 3 & $71,989,840$ & $87.9 \%$ & & & \\
\hline Liver1 & Liver & 1 & $69,832,472$ & $87.8 \%$ & & & \\
\hline Liver2 & Liver & 2 & $66,986,370$ & $86.9 \%$ & & & \\
\hline Liver3 & Liver & 3 & $75,826,328$ & $88.4 \%$ & & & \\
\hline Lung1 & Lung & 1 & $68,902,820$ & $85.8 \%$ & & & \\
\hline Lung2 & Lung & 2 & $71,797,208$ & $84.3 \%$ & & & \\
\hline Lung3 & Lung & 3 & $76,766,772$ & $84.7 \%$ & & & \\
\hline Muscle1 & Muscle & 1 & $72,955,650$ & $79.2 \%$ & $360,404,542$ & $87.2 \%$ & $85.9 \%$ \\
\hline Muscle2 & Muscle & 2 & $73,898,278$ & $81.7 \%$ & $476,837,820$ & $88.4 \%$ & $81.2 \%$ \\
\hline Muscle3 & Muscle & 3 & $79,972,012$ & $80.0 \%$ & $500,781,658$ & $88.8 \%$ & $82.1 \%$ \\
\hline Ovary1 & Ovary & 1 & $68,906,170$ & $85.6 \%$ & & & \\
\hline Ovary2 & Ovary & 2 & $86,181,796$ & $86.9 \%$ & & & \\
\hline Ovary3 & Ovary & 3 & $82,193,394$ & $86.3 \%$ & & & \\
\hline Average & & & $74,823,278$ & $85.6 \%$ & $446,008,007$ & $88.1 \%$ & $83.1 \%$ \\
\hline
\end{tabular}

${ }^{\mathrm{a}}$ The coverage was estimated based autosomal and $\mathrm{X}$ chromosomes

\section{RNA editing detection}

The detection of RNA editing sites was conducted using RES-Scanner [23]. Parameters were adopted according to the author's recommendation. The reference genome Sus scrofa 10.2.87 was downloaded from Ensembl (ftp:// ftp.ensembl.org/pub/release-87/fasta/sus_scrofa/dna/).

The genomic feature position files and Sus_scrofa.dbSNP145.gtf were prepared using custom scripts on the basis of Sus_scrofa.Sscrofa10.2.87.gtf (ftp://ftp.en sembl.org/pub/release-87/gtf/sus_scrofa/) and Sus_scrofa.vcf (ftp://ftp.ensembl.org/pub/release-87/variation/vcf/ sus_scrofa/) following the RES-Scanner user manual.

A candidate RNA editing site must meet the following conditions: 1) the genomic site is homozygous with a Bayesian probability exceeding 0.95 and is supported by at least 10 reads; 2) at least three RNA reads differ from the genomic genotype; 3 ) the site has an editing level $\geq 0.05$ and must be supported by at least one RNA read in the middle of its length; 4) the binomial test false discovery rate (FDR) of this site must be <0.05; and 5) the site is not located within homopolymeric regions of five or more residues and within six intronic bases of a splice site. All thresholds used for the identification of the RNA editing sites were the default parameters of RES-Scanner.

\section{Validation of RNA editing sites through sanger sequencing}

In total, 64 editing sites randomly selected from all sites identified in Brain3 were used to assess the reliability of RES-Scanner. RNA isolated from Brain3 was used for reverse transcription by a PrimeScript ${ }^{\mathrm{Tm}} \mathrm{RT}$ Reagent Kit (Takara, Japan) according to the manufacturer's instructions. DNA was extracted from Muscle3. The primers were designed by the National Center for Biotechnology Information (NCBI) Primer-BLAST and synthesized by Thermo Fisher Scientific Inc. (Beijing, China) to amplify appropriate fragments for Sanger sequencing (Additional file 2: Table S1). The sites were considered verified if the cDNA sequence was heterozygous while the corresponding DNA sequencing was homozygous.

\section{Gene expression and editing level}

To quantify the porcine gene expression level, Tophat2 [24] was applied with the default command options to align the RNA-seq reads against the reference genome 
(Sus scrofa 10.2.87). Then, HTseq [25] was used to count the reads aligned to each gene. Finally, fragments per kilobase million (FPKM), which is currently the most commonly used method for estimating gene expression levels [26], was calculated based on the length of the gene and the number of reads mapped to this gene. The RNA editing level at a given site was calculated as the ratio of reads supporting the edited base to the total number of reads covering the site. The editing level analysis was limited to RNA editing sites covered by at least 10 RNA reads.

\section{Functional enrichment analysis}

We used the clusterProfiler package [27] to conduct a functional enrichment analysis based on Gene Ontology (GO) biological processes and Kyoto Encyclopedia of Genes and Genomes (KEGG) pathway terms. Human gene sets were chosen as the background. GO/KEGG terms with $q$-value $<0.05$ were considered significantly enriched.

\section{Results}

\section{Identification of RNA editing sites in swine}

To accurately detect the candidate RNA editing sites at the transcriptome-wide level in swine, strand-specific poly(A)-positive RNA sequencing and matched DNA sequencing were performed using seven tissues from three pigs. After quality trimming, on average, 74.8 million reads were generated from each sample, with an average mapping rate of $85.6 \%$. Approximately $88.1 \%$ of the 1338 million pass-filter reads obtained from the DNA sequencing were successfully aligned to the Sus scrofa reference genome. A summary of the deep sequencing process is provided in Table 1.

To fully utilize our sequencing data, the possible RNA editing events were detected with RES-Scanner, which requires matched RNA-seq and DNA-seq data to rule out genomic single nucleotide variants and automatically separates the plus-strand alignments from the minus-strand alignments for the strand-specific RNA-seq libraries to identify the correct genomic loci of origin. Using this method, in total, 163315 RNA editing events at 74863 sites were detected within our datasets (edited sites in different tissues or animals were considered separate events), including 68934 A-to-G editing sites (Additional file 3: Table S2).

\section{Validation of predicted RNA editing sites}

First, we used an in silico approach as previously reported [28] to search for evidences of the detected RNA editing sites in porcine expressed sequence tags (ESTs) of NCBI. In brief, $50 \mathrm{bp}$ upstream and $50 \mathrm{bp}$ downstream of the flanking regions of the RNA editing sites were extracted and queried against the public pig ESTs using BLAST. Then, ESTs with an e-value $<10^{-5}$ were counted. Of the 74863 editing sites, 67450 (90.1\%) sites were covered by at least one EST sequence, and 45243 (60.4\%) sites were found in at least one RNA edited EST clone. The validation rates significantly varied across the different RNA editing types as follows: 63.7\% (43901/ 68934) of the A-to-G editing sites were supported by at least one RNA edited EST sequence, while less than 43\% of the RNA editing sites were validated for each of the 11 non-A-to-G types (range from 14.9\% to $42.5 \%$ ) (Fig. 1a). Then, Sanger sequencing was carried out to experimentally validate 64 editing sites (47 A-to-G sites and 17 non-A-to-G sites) across five genomic feature regions comprising CDS, noncoding RNA (ncRNA) and untranslated, intronic and intergenic regions. Forty-three A-to-G sites (91.4\%) and 10 other type sites (58.8\%), including two C-to-A, three G-to-A and five T-to-C sites, were experimentally verified (Fig. $1 \mathrm{~b}$ and Additional file 1: Figure S2). However, no known enzymes or biological mechanisms can catalyze the 10 non-A-to-G sites. Given these results, we focused on the A-to-G editing sites for further analysis.

\section{Characterization of porcine RNA editing sites}

In this study, 12 types of RNA editing were detected, including all possible base substitutions as follows: A-to-G, A-to-C, A-to-T, C-to-A, C-to-G, C-to-T, G-to-A, G-to-C, G-to-T, T-to-A, T-to-C and T-to-G (Fig. 2a). Overall, the A-to-G substitution was the most common type, accounting for up to $92.1 \%$ of the identified RNA editing sites. The editing level of the A-to-G sites was low overall, and $90 \%$ of the detected A-to-G sites had editing levels less than 55\% (Fig. 2b). We also observed that the identified A-to-G sites were widely and unevenly distributed across the Sus scrofa chromosomes (SSCs) as follows: more editing sites were detected on SSC1, SSC6 and SSC13 than on the other SSCs (Fig. 2c). The number of A-to-G sites had a similar tendency to change according to the length of the chromosomes. As expected, the number of RNA editing sites was significantly related to the chromosome length based on the correlation analysis $(r=0.82, P<0.01)$. Then, we ranked the chromosomes according to the difference between the normalized chromosome length (multiplying by the number of editing sites/the total length of chromosomes) and observed RNA editing sites. The top four chromosomes were SSC6, SSCX, SSC1 and SSC11. Given the large number of RNA editing sites, we were able to determine whether there was a sequence preference in the vicinity of the detected A-to-G sites. Consistent with the known attributes of ADAR substrates, the nucleotide immediately upstream of the editing site showed significantly depleted G, while the downstream nucleotide favored G (Fig. 2d). In addition, an avoidance of A was observed in the $5^{\prime}$ and 3' regions 


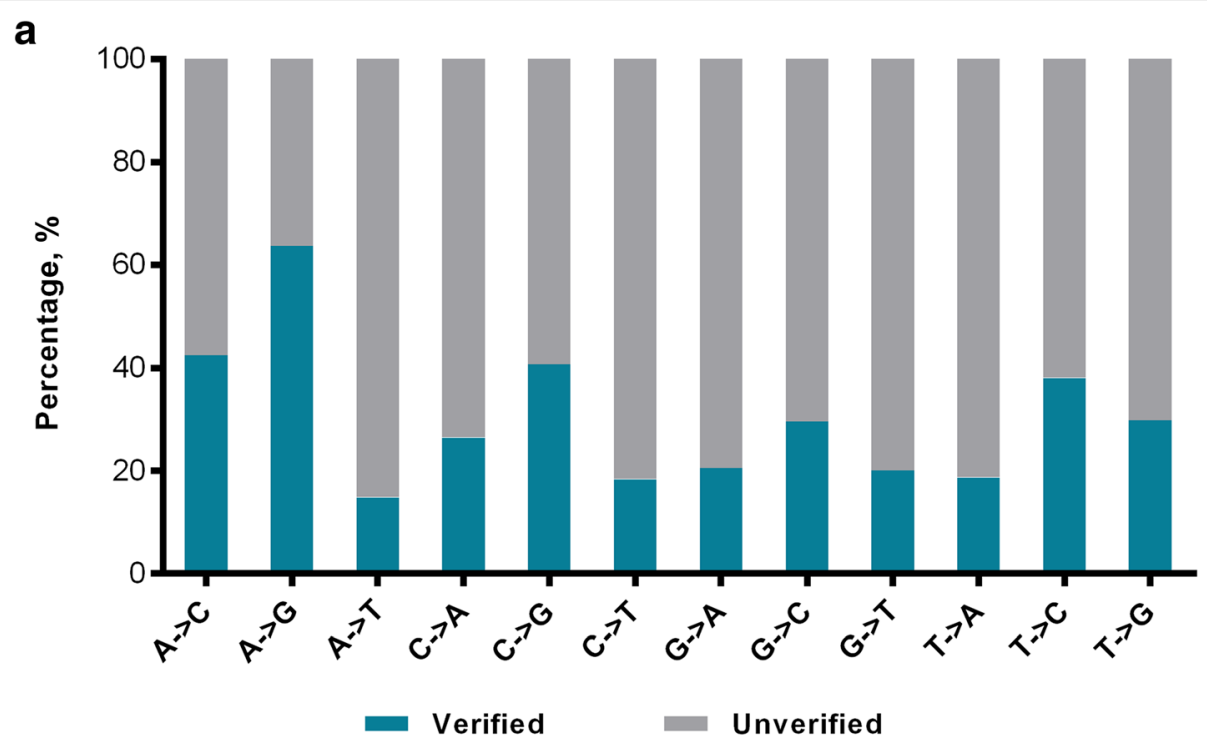

b

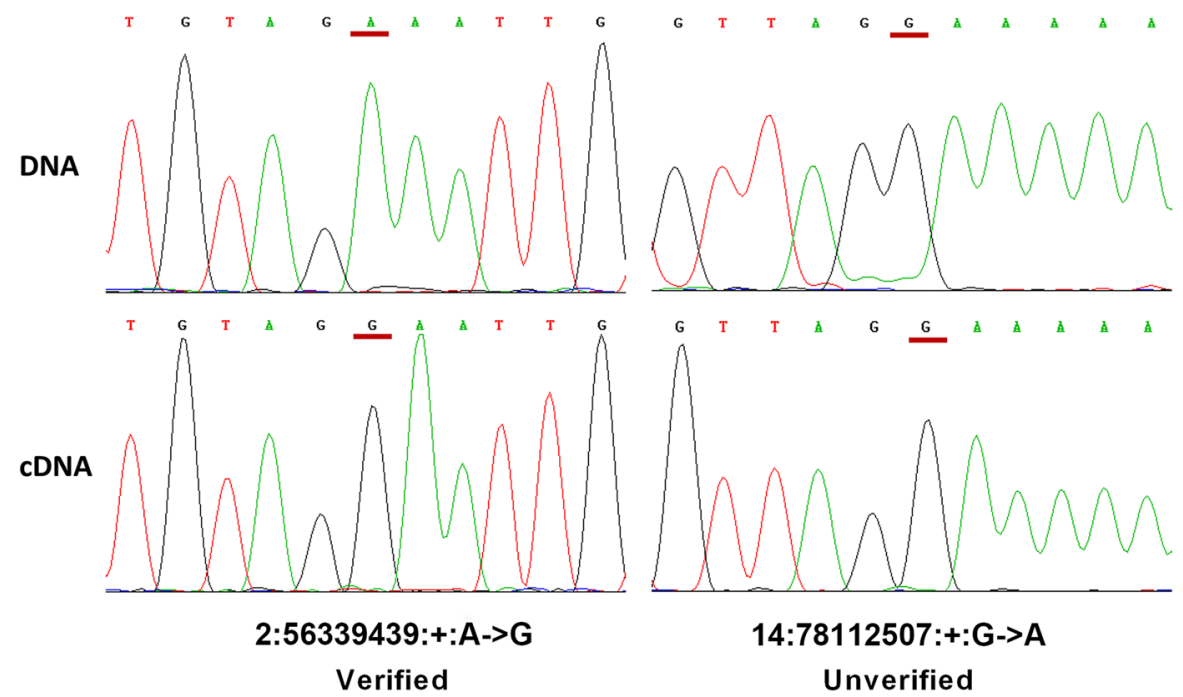

Fig. 1 Verification of RNA editing sites. a The validated rate of each RNA editing type by EST BLAST searching. A verified editing site means that the site is supported by at least one edited EST sequence. $\mathbf{b}$ An example showing the genotyping results of the genomic DNA and RNA of one verified RNA editing site (Chr2:56339439:+:A- > G) and one unverified RNA-editing site (Chr14:78112507:+:G- > A) by Sanger sequencing. The sites are highlighted in red lines

of the editing sites, which was also observed in the human inosinome [14] and rhesus macaque editome [5]. To explore whether porcine RNA editing sites also occurred in clusters similar to humans, the sites patterning in clusters $\geq 3$ sites within $100 \mathrm{bp}$ were calculated. We found that the extent of A-to-G site clustering widely ranged across the samples (from 24.8\% to 98.7\%, Additional file 4: Table S3). At the tissue level, the highest rate of A-to-G site clustering was found in the fat group ( $87.8 \%$ on average), while the lowest rate was found in the liver group (26.9\% on average). A cross-species comparative analysis was performed to compare our detected editing sites and the human editome retrieved from the REDIportal (http:// srv00.recas.ba.infn.it/atlas/) and DARNED (https://dar ned.ucc.ie/) databases. Using $50 \mathrm{bp}$ flanking regions of the porcine RNA editing sites, BLAST analyses were performed against $50 \mathrm{bp}$ flanking regions of the human sites. The sites supported by the ESTs with e-values <0.001, identity $>85 \%$ and alignment length $>50 \mathrm{bp}$ were considered conserved editing sites [28]. This analysis revealed 454 conserved A-to-G sites (Additional file 5: Table S4), which is comparable to a previous study [5]. 


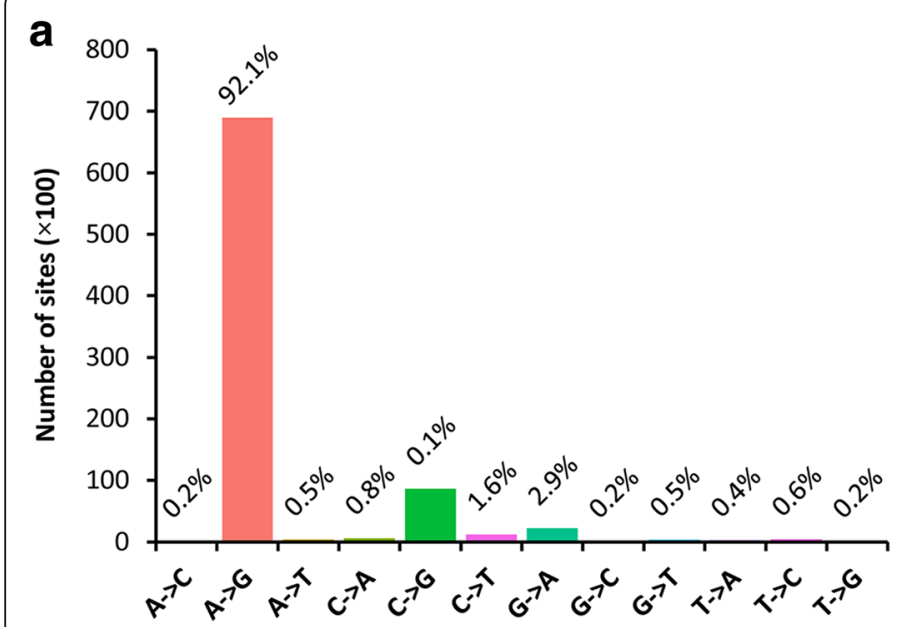

C

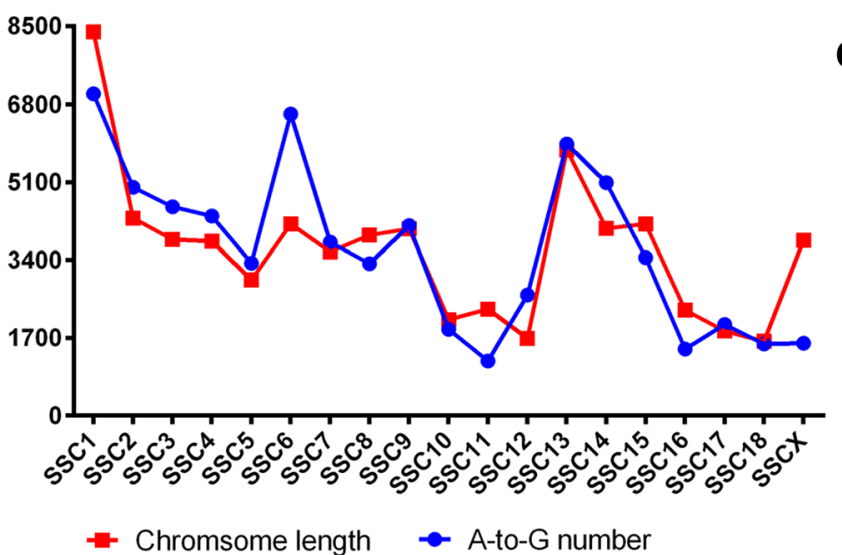

b

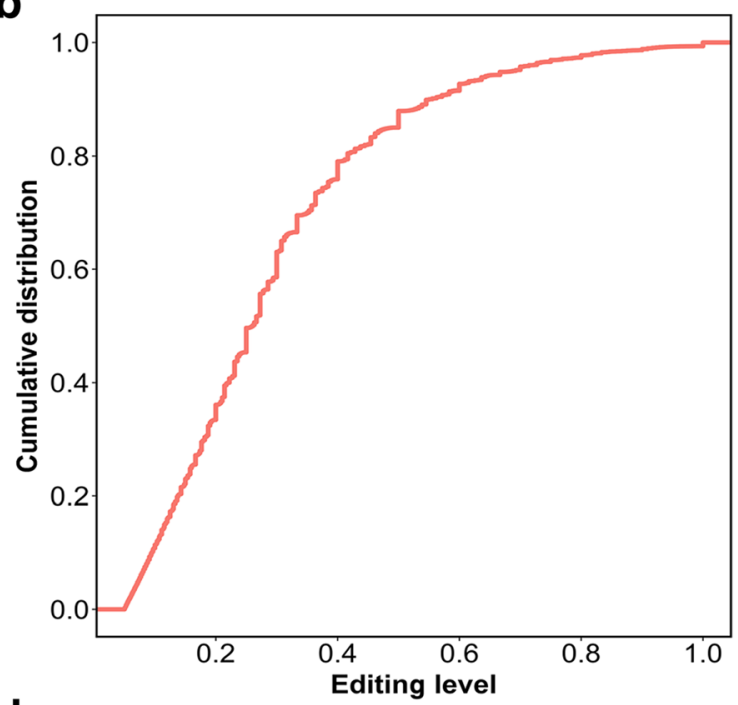

d

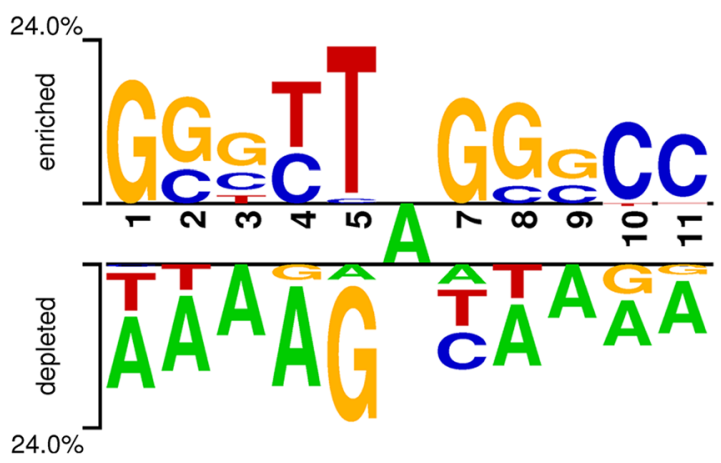

Fig. 2 Characteristics of the pig editome. a Distribution of RNA editing types. $\mathbf{b}$ Cumulative percentage distribution of the editing levels of A-to-G sites. The editing level of a given editing site is determined by the number of reads with the edited base divided by the total reads. If the same site was detected in multiple samples, the highest editing level was used in the analysis. c Chromosome distribution of A-to-G sites. Chromosome length was normalized by multiplying by the number of editing sites/the total length of chromosomes. $\mathbf{d}$ Sequence preference of A-to-G RNA editing sites. The enriched (above the top line) and depleted (below the bottom line) nucleotides near the focal editing sites are displayed in Two-Sample Logo. The height of the letters depicts the level of preference/depletion

\section{Analysis of the editing sites across genomic regions}

Next, we studied the location characteristics of the detected RNA editing sites, and the priority was consistent with ANNOVAR [29]. Interestingly, the largest fraction of A-to-G sites was located in intergenic regions, followed by introns (Fig. 3a). In total, 520 A-to-G sites were located in ncRNA, and most $(89.4 \%)$ of these sites occurred in introns. Less than $6 \%$ of the A-to-G sites were located in 3 ' untranslated regions (UTR), including 592 sites that overlapped with the target sites of miRNA seed regions $\left(2^{\text {nd }}\right.$ to $8^{\text {th }}$ nucleotides, the key region involved in the recognition between a miRNA and the 3' UTR of its target mRNA [30]) predicted by miRanda [31]. The top 20 miRNAs according to the number of edited targets are shown in Additional file 6: Table S5. Furthermore, we found that 341 of the remaining 3451 3’UTR sites potentially generated novel miRNA targets.
In addition, 151 A-to- $\mathrm{G}$ sites were detected in CDS, including 94 sites that could lead to nonsynonymous amino acid changes (Additional file 7: Table S6). Among the 94 missense sites, 30 sites had editing evidence in all seven studied tissues, and 59 sites were detected in at least two tissues. Notably, the top three most frequent substitution types, i.e., isoleucine to valine (I-to-V), threonine to alanine (T-to-A) and lysine to glutamate (K-to-E), accounted for more than $44 \%$ of all amino acid conversions (Fig. 3b). Up to $70.2 \%$ of the missense A-to-G sites were observed at the first or second position in codons. The putative impacts of the amino acid replacements predicted by snpEff [32] demonstrated that all 94 missense variances were likely to be moderate. Only four missense sites (Chr4:98044799, Chr7:102789222, 8:48244993 and Chr11:22178068) have been previously identified in a single female pig from an $F_{2}$ 


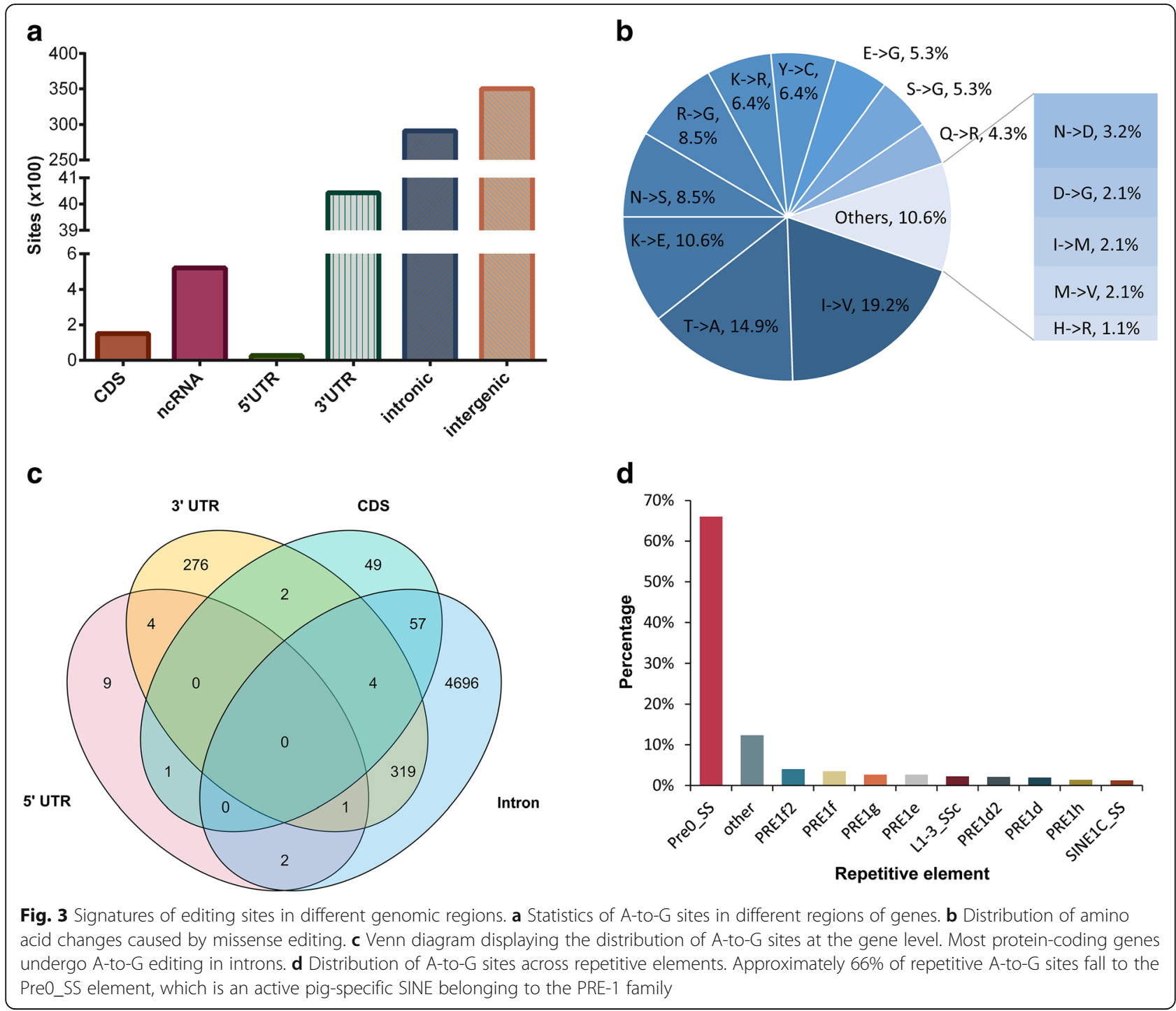

population [22]. This finding might be caused by a difference in breeds.

While concentrating on the distribution of A-to-G sites within a gene model, we found that $92.8 \%$ of the 5420 protein-coding genes undergoing RNA editing were edited in only one genic region (Fig. 3c). While 93.7\% of the edited genes had intronic editing sites, only $7.1 \%$ of the edited genes simultaneously had intronic and other genic region editing sites. The considerable intronic RNA editing sites and the observation that most genes were edited in intronic regions suggested that A-to-G editing may impact splicing as previously reported [33]. It is universally acknowledged that in primates, RNA editing sites are mostly located in Alu elements, which are categorized into short interspersed nuclear elements (SINEs). This property allowed us to understand the association between the RNA editome and repetitive elements in the pig. AnnotateTable.py from REDItools [34] was used to determine which repetitive elements contained the identified RNA editing sites. Consistent with previous studies [5, 22, 35], 97.3\% of the RNA editing sites located in the repeats were A-to-G conversions (Additional file 1: Figure S3), and 94.1\% of the repetitive A-to-G sites were located in SINEs (Additional file 1: Figure S4). By further subdividing the repetitive element families, we found that $88.6 \%$ of the repetitive A-to-G sites occurred within the PRE-1 family, and, notably, approximately $66 \%$ of these sites occurred in the Pre0_SS element, which is an active pig-specific SINE belonging to the PRE-1 family (Fig. 3d).

\section{Distribution of RNA editing sites across porcine tissues}

The number of A-to-G sites greatly varied across tissues and pigs (Fig. 4 and Additional file 1: Figure S5). Only 513 RNA editing sites spanning 169 expressed genes were shared by the seven tissues (Additional file 8: Table S7). 


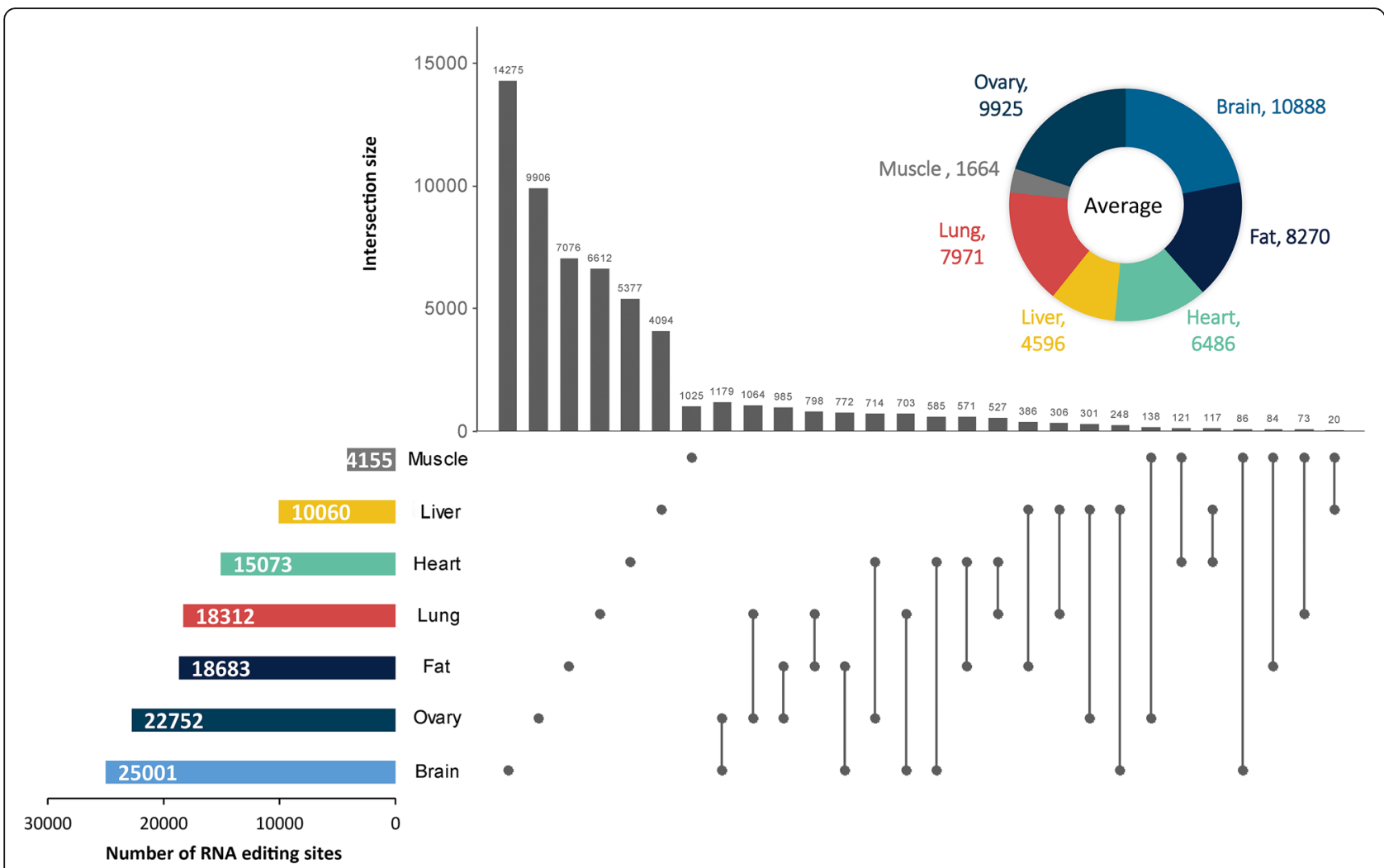

Fig. 4 Landscape of RNA editing sites across porcine tissues. The doughnut chart displays the average number of A-to-G sites in each tissue group. The horizontal bar chart displays the nonredundant A-to-G sites in each tissue group. The vertical bar chart shows the number of shared RNA editing sites across tissues

Less than $10 \%$ of the RNA editing sites were shared across the three samples of each tissue. These observations highlighted the variety and diversity of co/posttranscriptional modification. Overall, the brain was the most edited tissue, with an average of 10888 A-to-G sites, followed by the ovary, and the muscle had the least number of A-to-G sites (on average 1664). After removing the duplicates, the number of nonredundant RNA editing sites in the tissues ranged from 4155 to 25001 . The large number of sites in each tissue suggested that RNA editing is likely functionally important in nonbrain tissues. Unexpectedly, although an obvious difference was discovered in the number of A-to-G sites per sample, the detected sites exhibited strong comparability in editing levels, with the median level ranging from 0.2 to 0.25 (Fig. 5a). According to the hierarchical clustering analysis, we further found that the interindividual variations of the editing levels were smaller than the cross-tissue variation (Fig. 5b).

Interestingly, most tissue-shared sites were present in noncoding regions, especially the 3'UTR, and less than $6 \%$ of the shared sites were located in CDS. This result suggested that common RNA editing may function by regulating the expression of specific genes. While concentrating on the tissue specificity of RNA editing, we found that a host of A-to-G sites occurred only in specific tissues (Fig. 4). This finding highlighted the strong tissue specificity of RNA editing. Therefore, we further counted the tissue-specific sites by referring to a previously reported study [14]. Briefly, we mapped all detected A-to-G sites on the expressed genes with an FPKM $>1$ and then selected tissue-specific sites from the sites in the expressed genes. Unexpectedly, the ovary, rather than the brain, had the largest number of tissue-specific editing sites (Additional file 9: Table S8). Notably, less than $10 \%$ of the tissue-specific editing sites occurred in tissue-specific expressed genes within each tissue type. To characterize the functional significance of the tissue-specific editomes, a functional enrichment analysis of the genes with tissue-specific editing sites was carried out using the Bioconductor package clusterProfiler. As expected, these genes were significantly enriched in biological processes related to their respective tissue functions, such as "dendrite development" in the brain $\left(q\right.$-value $\left.=9.07 \times 10^{-5}\right)$, "lipid modification" in the fat $\left(q\right.$-value $\left.=4.98 \times 10^{-4}\right)$, "cardiac muscle cell differentiation" in the heart $\left(q\right.$-value $\left.=1.07 \times 10^{-3}\right)$, "carboxylic acid catabolic process" in the liver $\left(q\right.$-value $\left.=3.68 \times 10^{-14}\right)$, "vesicle organization" in the lung $\left(q\right.$-value $\left.=6.21 \times 10^{-3}\right)$, 

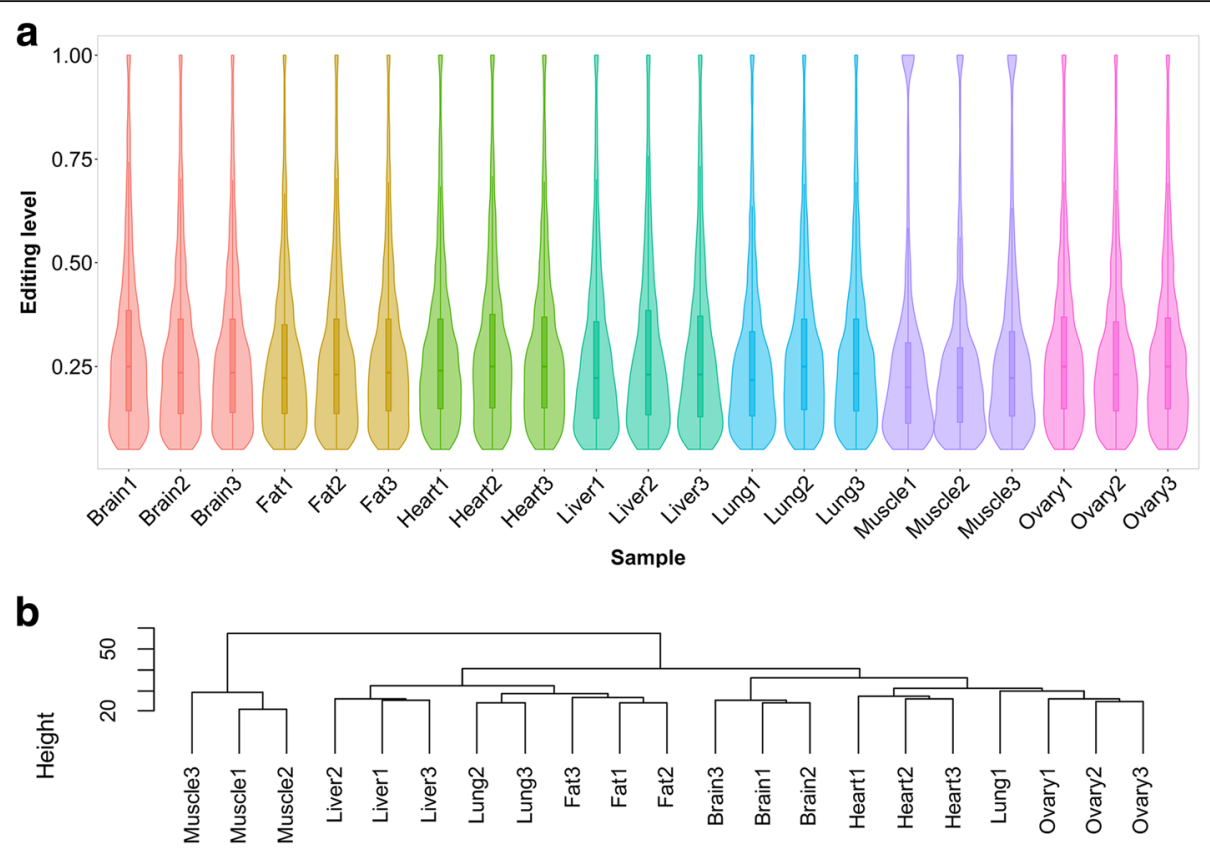

Fig. 5 Statistical features of RNA editing levels within and across samples. a The distribution of RNA editing levels across samples. Overall, the RNA editing levels are similar across tissues and within each tissue group. $\mathbf{b}$ Hierarchical clustering of RNA editing levels at all A-to-G sites across multiple tissues and individuals

"actomyosin structure organization" in the muscle $\left(q\right.$-value $\left.=3.28 \times 10^{-2}\right)$, and "cell cycle G2/M phase transition" in the ovary $\left(q\right.$-value $\left.=2.93 \times 10^{-8}\right)$. The top $5 \mathrm{GO}$ terms of each tissue according to the $q$-values are displayed in Additional file 1: Figure S6. Based on the pathway analysis (Fig. 6), we discovered obviously different enriched pathways among these tissues. In addition, most pathways enriched in each tissue were related to their respective tissue function, such as "glutamatergic synapse" in the brain $\left(q\right.$-value $\left.=4.33 \times 10^{-2}\right)$, "AMPK signaling pathway" in the fat $\left(q\right.$-value $\left.=3.64 \times 10^{-2}\right)$, and "fatty acid metabolism" $\left(q\right.$-value $\left.=1.21 \times 10^{-3}\right)$ in the liver. Specifically, more pathways were enriched in the fat and liver than in the other tissues, while no pathway was enriched in the muscle. Similar results were obtained even after excluding the tissue-specific expressed genes (Additional file 1: Figure S7).

\section{Association between ADAR expression levels and the porcine RNA editome}

It is well known that A-to-G editing is catalyzed by ADAR enzymes. Hence, we investigated whether the tissue differences in RNA editing are related to the differential expression of the $A D A R$ genes. First, we calculated the expression levels of the $A D A R 1, A D A R 2$ and $A D A R 3$ genes using our RNA-seq data. The total expression of the $A D A R \mathrm{~s}$ is the highest in the brain, and the lowest expression is in the muscle (Fig. 7). We also found that $A D A R 3$ was nearly exclusively expressed in the brain.
Next, we estimated the correlations between the expression of the $A D A R s$ and the RNA editing site number and between the expression of the ADARs (except for $A D A R 3$, which is only expressed in the brain) and the global RNA editing level (summing the editing levels at all positions) at the sample level. This analysis revealed a strong and statistically significant correlation between both the number of editing sites $(r=0.89, P<0.01)$ and the global level of editing $(r=0.89, P<0.01)$ with the expression of ADAR1 (Additional file 1: Figure S8). Statistically significant correlations were also observed for $A D A R 2$, but these correlations were not strong $(r=0.59$ for RNA editing number; $r=0.58$ for global RNA editing level). Then, by repeating this analysis at the tissue group level, statistically significant correlations were found only for $A D A R 1$ (number of editing sites: $r=0.96$, $P<0.01$; global level of editing: $r=0.95, \quad P<0.01$ ) (Additional file 1: Figure S9). These observations indicated that $A D A R 1$ may be the primary editor of the A-to-G sites.

\section{Discussion}

To accurately call the RNA editing sites, we meticulously designed our experiment. First, long paired-end reads were used to improve the genome mappability and facilitate the identification of hyperedited reads [36]. Second, strand-specific sequencing protocols were used to identify the correct genomic loci of origin while significantly controlling for potentially ambiguous calls due to 


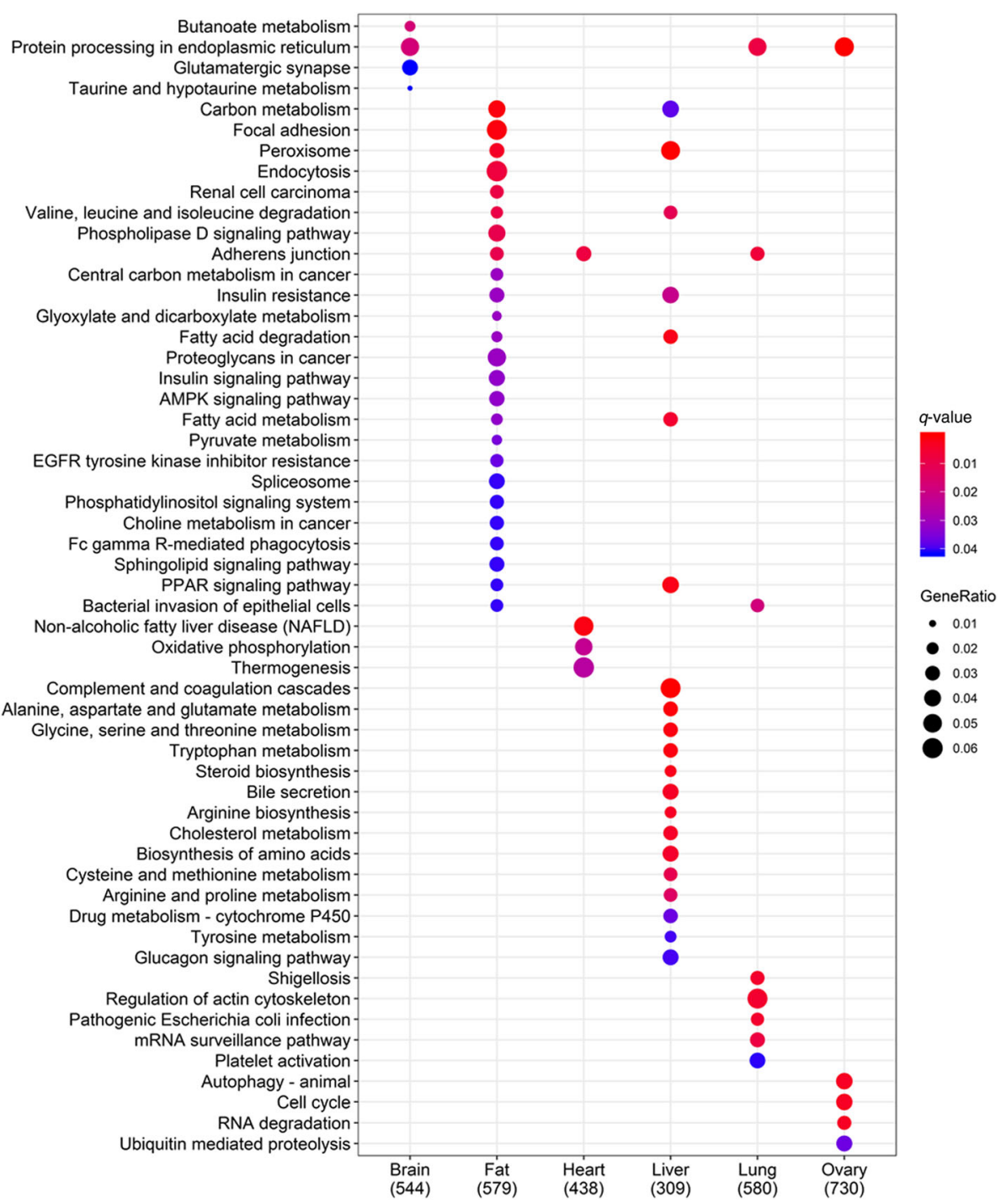

Fig. 6 Pathway enrichment analysis of the genes containing tissue-specific RNA editing. Dot plot of the enriched KEGG pathways in each tissue. Dot color indicates the statistical significance of the enrichment ( $q$-value); dot size represents the fraction of genes annotated to each term

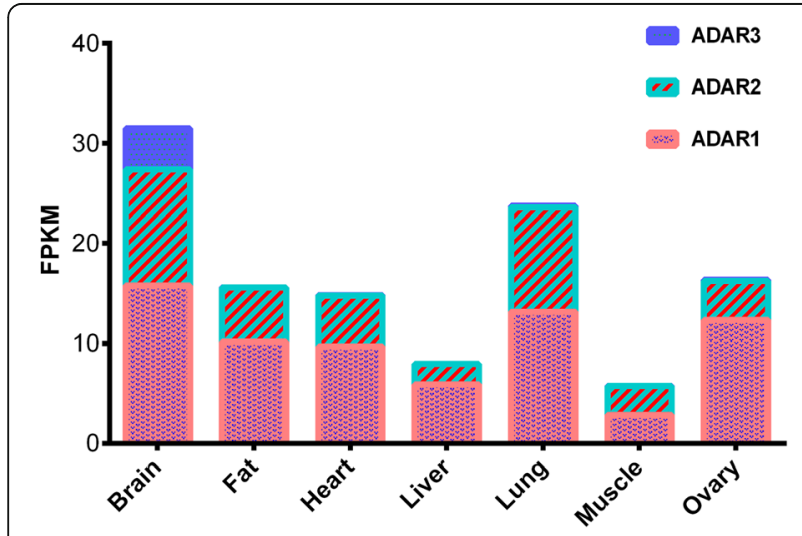

Fig. 7 Expression levels of ADAR genes across porcine tissues widespread anti-sense expression [5, 37]. Then, matched RNA and DNA sequencing was performed in porcine tissues from the same individuals, which efficiently eliminated genetic variations compared with working with RNA-seq data alone. Finally, RES-Scanner, which is an all-in-one tool that incorporates sophisticated statistical models, was applied to effectively distinguish the real RNA editing sites from potential false positives. These efforts ensured the accuracy and quantity of the editing site identification.

Overall, we identified 74863 editing sites, which is far less than that detected in a similar survey in humans (2013010 sites) [14]. A certain proportion of this difference could be attributed to the primate-specific Alu elements, which are active SINE retrotransposons. We further noted that there are more editing sites in pigs 
than in cows [28] and chickens [38]. The PRE-1 elements are pig-specific SINE retrotransposons that possess properties similar to Alu [39], and a previous study reported that RNA editing in swine is associated with PRE-1 elements [22]. Similar to the previous study, our results showed that $88.6 \%$ of the 67136 repetitive A-to-G sites occurred within the PRE-1 family. Hence, PRE-1 retrotransposons may contribute to the difference in the number of RNA editing sites between pigs and humans or other animals.

Consistent with previous studies in primates $[5,6,40]$, $>90 \%$ of the identified sites are of the A-to-G type, which is higher than that reported in the pig in a previous study (75\% A-to-G changes) [22]. This indicates the high accuracy of our detection referring to the study of Porath et al. [41]. Approximately $64 \%$ of the A-to-G sites were validated by at least one EST sequence, which is consistent with studies in mice ( $>55 \%$ of validation) [16] and cows (66\% of validation) [28]. To understand the mechanism of target recognition, the sequence contexts of the A-to-G editing sites were analyzed. The sequence preference in pigs is similar to that in humans [42], mice [43], cows [28] and chickens [38], indicating the conversion of the recognition mechanism. It is well known that A-to-G sites can be grouped into clusters and that an editing cluster increases the reliability of the contained editing sites. In the present study, we found strong tissue specificity of A-to-G site clustering.

In our research, the intergenic regions contained the largest number of RNA editing sites, which is different from humans, where intronic editing sites are the most common. A possible explanation for the difference is that the porcine genome annotation is of poor quality compared with the human annotation, and many unannotated genes are present (25322 pig genes vs 63305 human genes in Ensembl release 87). However, the abundance of RNA editing within the intronic and intergenic regions is unexpected because our RNA-seq libraries were purified using oligo(dT) beads to enrich polyadenylated mRNAs that had undergone splicing. This finding could be partially attributed to intron retention [44]. Moreover, SINEs may be another reason for this unexpected observation. SINEs are sequences of noncoding DNA that generally have more or less degenerate poly(A) tails [45]. It is entirely possible that a standard oligo $(\mathrm{dT})$ protocol introduces SINEs into RNA-seq libraries, increasing the reads that overlap with intronic and intergenic sequencing. We found 94 nonsynonymous A-to-G editing sites resulting in 15 amino acid change classes across 76 genes. Notably, a number of RNA editing sites that control $\mathrm{I} / \mathrm{V}, \mathrm{Y} / \mathrm{C}$ and $\mathrm{Q} / \mathrm{R}$ in GRIK2, I/V in COPA, I/V in KCNA1, T/A in ACOT4, R/G in GRIA2, $\mathrm{I} / \mathrm{V}, \mathrm{I} / \mathrm{M}$ and N/S in HTR2C and $\mathrm{R} / \mathrm{G}$ in GRIA3 have been identified in pigs in other studies $[22,46]$.
The three RNA editing sites in GRIK2 are considered important for regulating calcium permeability [47]. For COPA, I/V editing was detected in the current seven tissues, and its hypoediting is associated with hepatocellular carcinoma pathogenesis in humans [48]. The I/V recoding of KCNA1 via RNA editing affects the action potential shape, signal propagation and the firing pattern by accelerating the KCNA1 channel recovering from inactivation [49]. Consistent with previous work [22], ACOT4 was also found to be edited in fat, but the function of its editing is unknown. The $\mathrm{R} / \mathrm{G}$ recoding in GRIA2 and GRIA3 leads to faster desensitization recovery [50]. The RNA editing in HTR2C can reduce the efficacy of the interaction between receptors and their $G$ protein [51]. In addition to the above reported genes, we discovered several genes with nonsynonymous A-to-G editing sites for further studies investigating the function of RNA editing.

Although obvious variation was observed across the editing profiles of the samples, the intrapopulation variability in the editing levels is lower than that across tissues, suggesting a similarity to gene expression regulation [52]. By comparing the A-to-G editing profiles among seven porcine tissues, we discovered that most A-to-G sites were tissue-specific. However, 39.9\% of the hepatic A-to-G sites were common to adipose, which is comparable to previously reported findings [22]. In the functional enrichment study, we found that the tissue-specific editing site-containing genes were significantly enriched in pathways related to their respective tissue functions and that the enriched pathways obviously differed among these tissues. The glutamatergic synapse pathway is a major excitatory neurotransmission pathway in the mammalian central nervous system [53]. The AMPK signaling pathway can regulate adipose lipolysis and fat oxidation [54]. In fat, AMPK could directly phosphorylate lipases, such as hormone sensitive lipase and adipocyte triglyceride lipase $[55,56]$. The liver plays an important role in fatty acid metabolism [57]. These observations demonstrate that RNA editing may play an important role in porcine tissue regulation. Moreover, there were more enriched pathways in the fat and liver than in the other tissues. However, no pathway showed significant enrichment in the muscle. Consistent with this study, in mammals, fewer RNA editing sites have been reported in muscle than in other tissues $[5,14]$. In our study, we detected only 547 muscle-specific RNA editing sites that occurred in 410 expressed genes (FPKM $>1$ ). Of the 410 genes, 149 genes were mapped to KEGG pathways. Hence, the fewer genes compared to the gene numbers in other tissues (310 732 genes) might contribute to the observation that no pathway was enriched in the muscle. Our findings might provide a new layer of 
regulation underlying complex traits in pigs. Furthermore, RNA edits provide information that has been unexplored at the DNA level. Hence, RNA edits could be integrated with SNPs and used in genome selection to improve the accuracy of breeding. In addition, given that RNA editing is dynamically regulated, RNA editing sites could be used as markers to monitor development, health and response to feed in breeding.

The ADAR enzymes have been shown to be essential for normal life and development in mice $[8,9]$. Consistent with other mammals, three ADARs exist in pigs, including ADAR1, ADAR2 and ADAR3. Only ADAR1 and ADAR2 have been shown to be enzymatically active [58]. However, ADAR3 can inhibit RNA editing by competitively binding double stranded RNA [59]. Consistent with previous studies, $A D A R 1$ and $A D A R 2$ were highly expressed in the brain and lung, and ADAR3 was exclusively expressed in the brain $[5,14]$. Although the sample distribution of the A-to-G sites was significantly and positively correlated with the expression of the ADARs, the tendency to change did not perfectly align. This observation shows that the editing enzymes play an important role in RNA editing regulation but cannot explain all modification.

\section{Conclusions}

This study identified 74863 RNA editing sites using matched RNA and DNA sequencing data and revealed the comprehensive profile of RNA editing in pigs. We also provide further evidence supporting a previous observation that pig transcriptomes are highly editable in PRE-1 SINE elements. Furthermore, the functional analysis of the genes with tissue-specific editing sites in each tissue revealed the potentially functional importance of RNA editing in porcine tissue regulation. Our study largely extends the list of RNA editing sites in swine and provides deeper insight into the characteristics of the pig editome.

\section{Additional files}

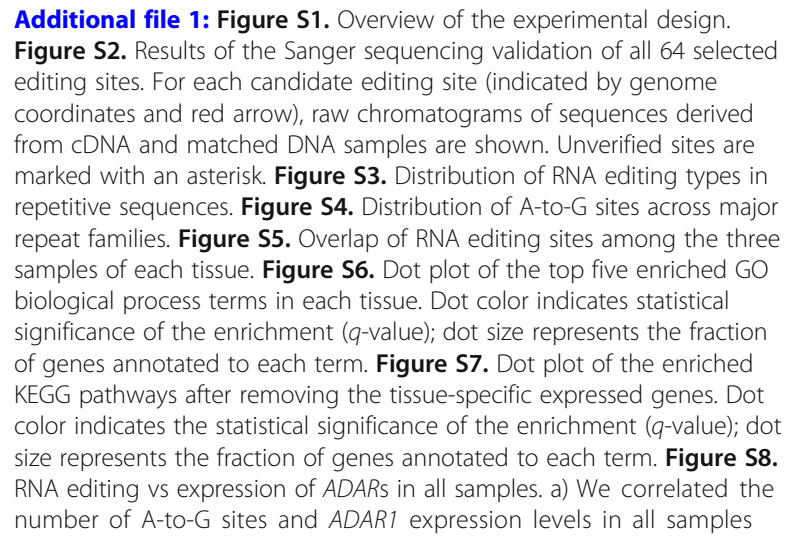

( $n=21)$. b) We correlated the number of A-to-G sites and ADAR2 expression levels in all samples $(n=21)$. c) and d) We calculated the correlations between the overall RNA editing levels and the expression values of ADAR1 and ADAR2 in all samples $(n=21)$. Correlation coefficients (r) and $P$-values are shown in each graph. Figure S9. RNA editing vs the expression of ADARs in tissue groups. a) We correlated the number of A-to-G sites and ADAR1 expression levels in all tissue groups $(n=7)$. b) We correlated the number of A-to-G sites and ADAR2 expression levels in all tissue groups $(n=7)$. c) and d) We calculated the correlations between the overall RNA editing levels and the expression values of ADAR1 and ADAR2 in tissue groups $(n=7)$. Correlation coefficients ( $r$ ) and $P$-values are displayed in each graph. (PDF $87057 \mathrm{~kb}$ )

Additional file 2: Table S1. Primers used in the Sanger sequencing. (XLSX $135 \mathrm{~kb}$ )

Additional file 3: Table S2. RNA editing sites identified in this study. (XLSX 6798 kb)

Additional file 4: Table S3. Clustering of A-to-G sites in each sample. (XLSX $9 \mathrm{~kb}$ )

Additional file 5: Table S4. Conserved A-to-G editing sites between pigs and humans. (XLSX 25 kb)

Additional file 6: Table S5. Top 20 miRNAs according to the number of edited targets. (XLSX 9 kb)

Additional file 7: Table S6. A-to-G sites resulting in amino acid changes. (XLSX $13 \mathrm{~kb}$ )

Additional file $\mathbf{8}$ Table S7. RNA editing sites shared by the seven examined tissues. (XLSX $27 \mathrm{~kb}$ )

Additional file $\mathbf{9}$ Table S8. Statistics of the tissue-specific A-to-G editing sites. (XLSX 9 kb)

\section{Abbreviations}

ADARs: Adenosine deaminases acting on RNA; A-to-G: Adenosine-toguanosine; CDS: Coding regions; EST: Expressed sequence tag; FPKM: Fragments per kilobase million; GO: Gene Ontology; KEGG: Kyoto Encyclopedia of Genes and Genomes; NCBI: National Center for Biotechnology Information; ncRNA: noncoding RNA; SINE: Short interspersed nuclear element; SSC: Sus scrofa chromosome; UTR: Untranslated regions

\section{Acknowledgements}

We thank the researchers at our laboratories for their dedication and hard work. We would like to thank everyone who made this thesis possible.

\section{Funding}

This research was supported by the National Key Technology R\&D Program of China (2015BAD03B02-2), Beijing Natural Science Foundation (6174047), earmarked fund for Modern Agro-industry Technology Research System (CARS-35), and Agricultural Science and Technology Innovation Program (ASTIP-IAS02).

\section{Availability of data and materials}

The sequencing data used in the current study are available at the Sequence Read Archive, BioProject PRJNA493166 (http://www.ncbi.nlm.nih.gov/bioproject/493166).

\section{Authors' contributions}

ZYB carried out the experiment and analysis. ZYB drafted the manuscript. ZYB, ZLC, WLG and WLX conceived and designed the experiments. All authors read and approved the final manuscript.

\section{Ethics approval and consent to participate}

All animals were sacrificed by electric shock in Beijing Fifth Meat Processing Factory according to the guidelines for experimental animals established by the Council of China. The experiments involving animals were approved by the Science Research Department of the Institute of Animal Science, Chinese Academy of Agricultural Sciences (CAAS) (Beijing, China).

\section{Consent for publication}

Not applicable. 


\section{Competing interests}

The authors declare that they have no competing interests.

Received: 27 September 2018 Accepted: 24 January 2019 Published online: 13 March 2019

\section{References}

1. Benne R, Van Den Burg J, Brakenhoff JP, Sloof P, Van Boom JH, Tromp MC Major transcript of the frameshifted coxll gene from trypanosome mitochondria contains four nucleotides that are not encoded in the DNA. Cell. 1986:46:819-26.

2. Farajollahi S, Maas S. Molecular diversity through RNA editing: a balancing act. Trends Genet. 2010;26:221-30.

3. Tajaddod M, Jantsch MF, Licht K. The dynamic epitranscriptome: a to I editing modulates genetic information. Chromosoma. 2016:125:51-63.

4. Nishikura K. A-to-l editing of coding and non-coding RNAs by ADARs. Nat Rev Mol Cell Biol. 2016;17:83-96.

5. Chen J, Peng Z, Zhang R, Yang X, Tan BC, Fang H, et al. RNA editome in rhesus macaque shaped by purifying selection. PLoS Genet. 2014;10: e1004274

6. Peng Z, Cheng Y, Tan BC-M, Kang L, Tian Z, Zhu Y, et al. Comprehensive analysis of RNA-Seq data reveals extensive RNA editing in a human transcriptome. Nat Biotechnol. 2012;30:253-60.

7. Valente L, Nishikura K. ADAR gene family and A-to-I RNA editing: diverse roles in posttranscriptional gene regulation. Prog Nucleic Acid Res Mol Biol. 2005:79:299-338.

8. Higuchi M, Maas S, Single FN, Hartner J, Rozov A, Burnashev N, et al. Point mutation in an AMPA receptor gene rescues lethality in mice deficientin the RNA-editing enzyme ADAR2. Nature. 2000;406:78-81.

9. Wang Q, Khillan J, Gadue P, Nishikura K. Requirement of the RNA editing deaminase ADAR1 gene for embryonic erythropoiesis. Science. 2000;290: 1765-8.

10. Brennicke A, Marchfelder A, Binder S. RNA editing. FEMS Microbiol Rev. 1999:23:297-316

11. Wang Y, Xu X, Yu S, Jeong KJ, Zhou Z, Han L, et al. Systematic characterization of A-to-I RNA editing hotspots in microRNAs across human cancers. Genome Res. 2017:27:1112-25.

12. Wang C, Zou J, Ma X, Wang E, Peng G. Mechanisms and implications of ADAR-mediated RNA editing in cancer. Cancer Lett. 2017:411:27-34.

13. Tan MH, Li Q, Shanmugam R, Piskol R, Kohler J, Young AN, et al. Dynamic landscape and regulation of RNA editing in mammals. Nature. 2017;550: 249-54.

14. Picardi E, Manzari C, Mastropasqua F, Aiello I, D'Erchia AM, Pesole G. Profiling RNA editing in human tissues: towards the inosinome atlas. Sci Rep. 2015;5:14941

15. Blanc V, Park E, Schaefer S, Miller M, Lin Y, Kennedy S, et al. Genome-wide identification and functional analysis of Apobec-1-mediated C-to-U RNA editing in mouse small intestine and liver. Genome Biol. 2014;15:R79.

16. Lagarrigue S, Hormozdiari F, Martin $\sqcup$, Lecerf F, Hasin Y, Rau C, et al. Limited RNA editing in exons of mouse liver and adipose. Genetics. 2013; 193:1107-15.

17. Gu T, Buaas FW, Simons AK, Ackert-Bicknell CL, Braun RE, Hibbs MA Canonical A-to-I and C-to-U RNA editing is enriched at 3'UTRs and microRNA target sites in multiple mouse tissues. PLoS One. 2012;7:e33720.

18. Danecek $P$, Nellaker C, McIntyre RE, Buendia-Buendia JE, Bumpstead $S$ Ponting CP, et al. High levels of RNA-editing site conservation amongst 15 laboratory mouse strains. Genome Biol. 2012;13:26.

19. Li M, Wang IX, Li Y, Bruzel A, Richards AL, Toung JM, et al. Widespread RNA and DNA sequence differences in the human transcriptome. Science. 2011; 333:53-8.

20. Paz N, Levanon EY, Amariglio N, Heimberger AB, Ram Z, Constantini S, et al. Altered adenosine-to-inosine RNA editing in human cancer. Genome Res. 2007;17:1586-95

21. Gallo A, Locatelli F. ADARs: allies or enemies? The importance of A-to-I RNA editing in human disease: from cancer to HIV-1. Biol Rev. 2012:87:95-110.

22. Funkhouser SA, Steibel JP, Bates RO, Raney NE, Schenk D, Ernst CW. Evidence for transcriptome-wide RNA editing among Sus scrofa PRE-1 SINE elements. BMC Genomics. 2017:18:360.

23. Wang Z, Lian J, Li Q, Zhang P, Zhou Y, Zhan X, et al. RES-scanner: a software package for genome-wide identification of RNA-editing sites. GigaScience. 2016;5:37.
24. Kim D, Pertea G, Trapnell C, Pimentel H, Kelley R, Salzberg SL. TopHat2: accurate alignment of transcriptomes in the presence of insertions, deletions and gene fusions. Genome Biol. 2013;14:R36.

25. Anders S, Pyl PT, Huber W. HTSeq-a Python framework to work with highthroughput sequencing data. Bioinformatics. 2015; 31:166-169

26. Trapnell C, Williams BA, Pertea G, Mortazavi A, Kwan G, Van Baren MJ, et al. Transcript assembly and quantification by RNA-Seq reveals unannotated transcripts and isoform switching during cell differentiation. Nat Biotechnol. 2010;28:511.

27. Yu G, Wang L, Han Y, He Q. clusterProfiler: an R package for comparing biological themes among gene clusters. OMICS: J Integrative Biol. 2012;16: 284-7.

28. Bakhtiarizadeh MR, Salehi A, Rivera RM. Genome-wide identification and analysis of A-to-I RNA editing events in bovine by transcriptome sequencing. PLoS One. 2018;13:e0193316.

29. Wang K, Li M, Hakonarson H. ANNOVAR: functional annotation of genetic variants from high-throughput sequencing data. Nucleic Acids Res. 2010;38:e164.

30. Lambert NJ, Gu SG, Zahler AM. The conformation of microRNA seed regions in native microRNPs is prearranged for presentation to mRNA targets. Nucleic Acids Res. 2011;39:4827-35

31. Betel D, Wilson M, Gabow A, Marks DS, Sander C. The microRNA. Org resource: targets and expression. Nucleic Acids Res. 2008;36:D149-D53.

32. Cingolani $P$, Platts $A$, Wang $L L$, Coon $M$, Nguyen $T$, Wang $L$, et al. A program for annotating and predicting the effects of single nucleotide polymorphisms, SnpEff: SNPs in the genome of Drosophila melanogaster strain w1118; iso-2; iso-3. Fly. 2012;6:80-92.

33. Goldberg L, Abutbul-Amitai M, Paret G, Nevo-Caspi Y. Alternative splicing of STAT3 is affected by RNA editing. DNA Cell Biol. 2017:36:367-76.

34. Picardi E, Pesole G. REDltools: high-throughput RNA editing detection made easy. Bioinformatics. 2013;29:1813-4.

35. Ramaswami G, Zhang R, Piskol R, Keegan LP, Deng P, O'connell MA, et al. Identifying RNA editing sites using RNA sequencing data alone. Nat Methods. 2013;10:128.

36. Carmi S, Borukhov I, Levanon EY, Identification of widespread ultra-edited human RNAs. PLoS Genet. 2011;7:e1002317.

37. Bass B, Hundley H, Li JB, Peng Z, Pickrell J, Xiao XG, et al. The difficult calls in RNA editing. Nat Biotechnol. 2012;30:1207.

38. Bakhtiarizadeh MR, Shafiei $H$, Salehi A. Large-scale RNA editing profiling in different adult chicken tissues. bioRxiv. 2018:319871.

39. Yu H, Wu Q, Zhang J, Zhang Y, Lu C, Cheng Y, et al. Genome-wide characterization of PRE-1 reveals a hidden evolutionary relationship between suidae and primates. BioRxiv. 2015:025791.

40. Ramaswami G, Lin W, Piskol R, Tan MH, Davis C, Li JB. Accurate identification of human Alu and non-Alu RNA editing sites. Nat Methods. 2012;9:579.

41. Porath HT, Carmi S, Levanon EY. A genome-wide map of hyper-edited RNA reveals numerous new sites. Nat Commun. 2014;5:4726.

42. Bazak L, Haviv A, Barak M, Jacobhirsch J, Deng P, Zhang R, et al. A-to-I RNA editing occurs at over a hundred million genomic sites, located in a majority of human genes. Genome Res. 2014;24:365.

43. Huntley MA, Lou M, Goldstein LD, Lawrence M, Dijkgraaf GJP, Kaminker JS, et al. Complex regulation of ADAR-mediated RNA-editing across tissues. BMC Genomics. 2016;17:61.

44. Rodriguez J, Menet JS, Rosbash M. Nascent-seq indicates widespread cotranscriptional rna editing in drosophila. Mol Cell. 2012;47:27-37.

45. Moran C. Molecular Genetics. In: F.Rothschild M, Ruvinsky A, editors. The genetics of the pig. UK: CABl; 2011. p. 73-100.

46. Venø M, Bramsen JB, Bendixen C, Panitz F, Holm I, Öhman M, et al. Spatiotemporal regulation of ADAR editing during development in porcine neural tissues. RNA Biol. 2012;9:1054-65.

47. Köhler M, Burnashev N, Sakmann B, Seeburg PH. Determinants of Ca2+ permeability in both TM1 and TM2 of high affinity kainate receptor channels: diversity by RNA editing. Neuron. 1993;10:491-500.

48. Chan THM, Lin CH, Qi L, Fei J, Li Y, Yong KJ, et al. A disrupted RNA editing balance mediated by ADARs (adenosine DeAminases that act on RNA) in human hepatocellular carcinoma. Gut. 2014;63:832-43.

49. Gonzalez C, Lopez-Rodriguez A, Srikumar D, Rosenthal JJ, Holmgren M. Editing of human KV 1.1 channel mRNAs disrupts binding of the Nterminus tip at the intracellular cavity. Nat Commun. 2011:2:436.

50. Lomeli H, Mosbacher J, Melcher T, Hoger T, Kuner T, Monyer H, et al. Control of kinetic properties of AMPA receptor channels by nuclear RNA editing. Science. 1994;266:1709-13. 
51. Burns CM, Chu H, Rueter SM, Hutchinson LK, Canton H, Sanders-Bush E, et al. Regulation of serotonin-2C receptor G-protein coupling by RNA editing. Nature. 1997;387:303.

52. Brawand D, Soumillon M, Necsulea A, Julien P, Csárdi G, Harrigan P, et al. The evolution of gene expression levels in mammalian organs. Nature. 2011:478:343.

53. Shin Y, H-j J, Jung M, Yoo S, Subramaniyam S, Markkandan $K$, et al. Discovery of gene sources for economic traits in Hanwoo by wholegenome resequencing. Asian Australas J Anim Sci. 2016;29:1353.

54. Yu H, Zhang M, Ma Y, Lu J, Pan J, Pan P, et al. 5-ALA ameliorates hepatic steatosis through AMPK signaling pathway. J Mol Endocrinol. 2017;59:121-8.

55. Watt MJ, Holmes AG, Pinnamaneni SK, Garnham AP, Steinberg GR, Kemp BE, et al. Regulation of HSL serine phosphorylation in skeletal muscle and adipose tissue. American Journal of Physiology-Endocrinology and Metabolism. 2006;290:E500-E8

56. Ahmadian M, Abbott MJ, Tang T, Hudak CS, Kim Y, Bruss M, et al. Desnutrin/ ATGL is regulated by AMPK and is required for a brown adipose phenotype. Cell Metab. 2011;13:739-48.

57. Frayn KN, Arner P, Ykijärvinen H. Fatty acid metabolism in adipose tissue, muscle and liver in health and disease. Essays Biochem. 2006;42:89.

58. Nishikura K. Functions and regulation of RNA editing by ADAR deaminases. Annu Rev Biochem. 2010:79:321-49.

59. Oakes E, Anderson A, Cohen-Gadol A, Hundley HA. Adenosine deaminase that acts on RNA 3 (ADAR3) binding to glutamate receptor subunit $B$ premRNA inhibits RNA editing in glioblastoma. J Biol Chem. 2017;292:4326.

Ready to submit your research? Choose BMC and benefit from:

- fast, convenient online submission

- thorough peer review by experienced researchers in your field

- rapid publication on acceptance

- support for research data, including large and complex data types

- gold Open Access which fosters wider collaboration and increased citations

- maximum visibility for your research: over $100 \mathrm{M}$ website views per year

At $\mathrm{BMC}$, research is always in progress.

Learn more biomedcentral.com/submissions 